\title{
Modeling of chemical composition in the melt pool during laser welding of Aluminum / Magnesium alloys
}

Sabrine Ben Halim ${ }^{1 *}$, Wassim kriaa $^{1}$ and Michel Autric ${ }^{2}$

${ }^{1}$ Laboratoire de Thermique et Thermodynamique des Procédés Industriels, Ecole Nationale d'Ingénieurs, Monastir, Tunisia

${ }^{2}$ Aix-Marseille University, CNRS, IUSTI, Marseille, France

\begin{abstract}
Joining dissimilar metals is very difficult due to the formation of brittle intermetallic phases which may be detrimental to mechanical properties. The present work aims to investigate the transport phenomenon in the weld bead and to understand the materials mixing during laser welding process of dissimilar AluminumMagnesium alloys. A three-dimensional transient model based on fluid flow, heat and mass transfer has been developed to predict the formation of the weld and to study numerically and experimentally the diffusion of alloying elements in the melted zone. SEM analysis of chemical composition has been realized to map elements distribution in the melted zone. The results of simulation show the formation of a heterogeneous mixture in the melt pool. The elements distribution map and the presence of brittle intermetallic phases in the fusion zone were analysed. The formation of intermetallic compounds, comprising $\mathrm{Al}_{3} \mathrm{Mg}_{2}$ and $\mathrm{Al}_{12} \mathrm{Mg}_{17}$ phases were predicted by studying the chemical elements distribution in the weld pool. A good tendency between experimental and numerical results is noticed for the weld.
\end{abstract}




\section{Introduction}

Laser welding is considered as one of the most effective process for joining different materials. It has been used for several years to join alloys and thin automotive panels because of its high energy density, high welding speed and narrow heat-affected zone [1]. In the bibliography, there are several research works on welding of dissimilar materials but the works on heterogeneous aluminum-magnesium joining are limited. Dehghani et al. [2] performed welding of AA5186 aluminum alloy and low carbon steel by FSW. They determined the effects of various parameters of the arc welding process, such as tool traverse speed and angle of inclination on the intermetallic volume fraction and the mechanical properties of the joint. The laser brazing welding was performed by Chen et al. [3]. They studied experimentally and numerically the titanium-aluminum joining. An inhomogeneous mixture is formed during the welding process. G. R. Mohammed et al. [4] studied the pulsed laser welding of stainless steel (304) and austenitic duplex (2205). They highlighted the effect of laser beam diameter and pulsed laser power, on the weld bead geometry. The increase in laser power leads to an increase in width and depth. Z. Liang et al. [5] evaluated the weldability of aluminum and magnesium by stir friction. They conducted that brittle intermetallic compounds (IMCs), such as $\gamma-\mathrm{Al}_{12} \mathrm{Mg}_{17}$ and $\beta-\mathrm{Al}_{3} \mathrm{Mg}_{2}$, were produced at the interfaces resulting in the formation of microcracks. G. Virgil et al. [6] reported the joining of Magnesium to aluminum by laser. A complex vortex flow in the weld pool and a large number of intermetallic phases were formed. In this study, a three-dimensional model is proposed to study the dissimilar metal aluminum to magnesium laser welding process and weld bead formation. The predicted elements distribution in the melting zone based on the model is validated by comparing the simulation results with experimental ones

\section{Experimental Procedure}

In this modeling work, the Aluminum alloy (A5754) and the Magnesium alloy (AZ31) were used as base materials and were arranged in a butt configuration, figure 1. The experiments were carried out with a continuous $\mathrm{Yb}$ : YAG laser with a wavelength of 1030 $\mathrm{nm}$. The spot size of the laser beam on the workpieces was $0.4 \mathrm{~mm}$ with a Gaussian intensity distribution [7]. The laser welding was at $2500 \mathrm{~W}$. An Argon shielding gas with a flow rate of $201 /$ min was used to protect the weld bead from oxidation.

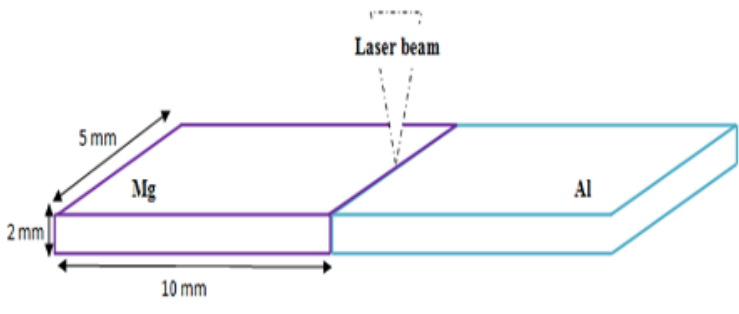

Fig. 1. Schematic of the laser spot welding process.

\section{Analysis And Modelling}

Heat transfer, fluid flow, and mass transport during Laser butt welding of dissimilar couple Aluminum / Magnesium were simulated by 3D transient numerical model developed using FLUENT software. The domain used for modeling is $20 \times 5 \times 2 \mathrm{~mm}^{3}$ (length $\times$ width $\times$ thickness). The calculation was carried out with a mesh composed of 400,000 cells. A uniform mesh over the entire area of computation was adopted.

The assumptions adopted were made:

-The thermo-physical properties vary with temperature and composition.

-Fluid flow in the weld pool is incompressible and laminar.

-The model is unsteady.

-The free surface of the weld pool is flat.

-The workpiece moves with a constant speed $\mathrm{U}_{\mathrm{y}}$.

\section{Equations}

This study is based on the resolution of conservation equations presented as follows [8]:

Mass conservation

$\frac{\partial(\rho)}{\partial t}+\nabla \cdot(\rho \vec{v})=0$

Where $\vec{v}$ is the speed following the respective directions.

Momentum conservation

$\frac{\partial(\rho \vec{v})}{\partial t}+\nabla \cdot(\rho \vec{v} \vec{v})=-\nabla p+\nabla \cdot(\mu \nabla \vec{v})+\rho \vec{g}+\vec{S}$

Where $\mathrm{p}$ is the pressure, $\mu$ is the viscosity and $\vec{S}$ is the source term.

Energy conservation

$\frac{\partial(\rho H)}{\partial t}+\nabla \cdot(\rho \vec{v} H)=\nabla \cdot(k \nabla T)+S_{E}$ 
Where $\mathrm{H}$ is enthalpy and $\mathrm{S}_{\mathrm{E}}$ is the energy source term.

Species conservation

$$
\frac{\partial(\rho C)}{\partial t}+\nabla \cdot(\rho \vec{v} C)=\nabla \cdot(\rho D \nabla C)
$$

Where $\mathrm{C}$ is the concentration and $\mathrm{D}$ is the mass diffusivity.

The heat transfer and fluid flow models were coupled with a melting and solidification model. Melting and solidification of the weld pool was simulated with an enthalpy-porosity formulation. It indicates the liquid fraction in the welding pool. The mushy region (partially solidified region) was treated as a porous medium.

The momentum sink due to the reduced porosity in the mushy zone can be expressed as:

$\overrightarrow{\mathrm{S}}=-\left(\frac{\mathrm{A}(1-\beta)^{2}}{\beta^{3}+\mathrm{b}}\right) \overrightarrow{\mathrm{v}}$

Where $\varepsilon$ is the liquid fraction, A is the mushy zone constant (a large number, say $10^{8}$ ) and $b$ is an arbitrary small number to prevent division by zero $\left(10^{-3}\right)$.

\section{Boundary and initial Conditions}

At time $\mathrm{t}=0$, the entire domain was considered to be in the solid state with a temperature of $300 \mathrm{~K}$ and the gravitational acceleration was assumed to be 9.81 $\mathrm{m} . \mathrm{s}^{-2}$,

At the flat free surface of the liquid, shear force due to surface tension (Marangoni force) is expressed as:

$\boldsymbol{\mu} \frac{\mathrm{du}}{\mathrm{dz}}=\boldsymbol{\beta} \frac{\partial \boldsymbol{\sigma}}{\partial \mathrm{T}} \frac{\partial \mathrm{T}}{\partial \mathrm{x}}+\boldsymbol{\beta} \frac{\partial \boldsymbol{\sigma}}{\partial C} \frac{\partial \mathrm{C}}{\partial \mathrm{x}}$

$\mu \frac{\mathrm{dv}}{\mathrm{dz}}=\beta \frac{\partial \sigma}{\partial \mathrm{T}} \frac{\partial \mathrm{T}}{\partial \mathrm{y}}+\beta \frac{\partial \sigma}{\partial C} \frac{\partial \mathrm{C}}{\partial \mathrm{y}}$

Where $\sigma_{\mathrm{T}}$ is thermal surface tension coefficient.

The heat loss by convection heat transfer and radiation is given by,

$$
\begin{aligned}
& \mathrm{q}_{\mathrm{rad}}=\varepsilon \sigma\left(\mathrm{T}^{4}-\mathrm{T}_{0}^{4}\right) \\
& \mathrm{q}_{\text {conv }}=\mathrm{h}_{\text {conv }}\left(\mathrm{T}-\mathrm{T}_{0}\right)
\end{aligned}
$$

Where $h_{\text {conv }}$ is the convection coefficient, $\sigma$ is the Stefan-Boltzmann constant and $\varepsilon$ is the emissivity of the surface radiation.

\section{Results and discussion}

Figure 2 shows the experimental and numerical profiles of the mass fraction of aluminum and magnesium at the top of the weld bead. It can be seen that the magnesium concentration obtained numerically decreases from $100 \%$ ( $\mathrm{Mg}$ base metal) to a value of about $70 \%$ in the molten zone while the aluminum concentration increases to $25 \%$ in the molten zone.

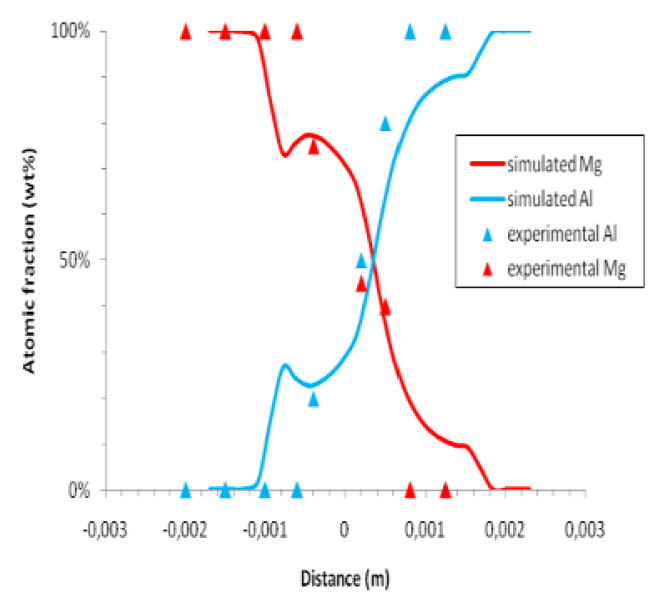

Fig. 2. Distribution of chemical elements at the top of the weld.

Figure 3 shows that the aluminum concentration increases from $0 \%$ (base metal $\mathrm{Mg}$ ) to a value of about $35 \%$ in the melting zone while the magnesium concentration decreases to a value of about $65 \%$ by mass in the melting zone.

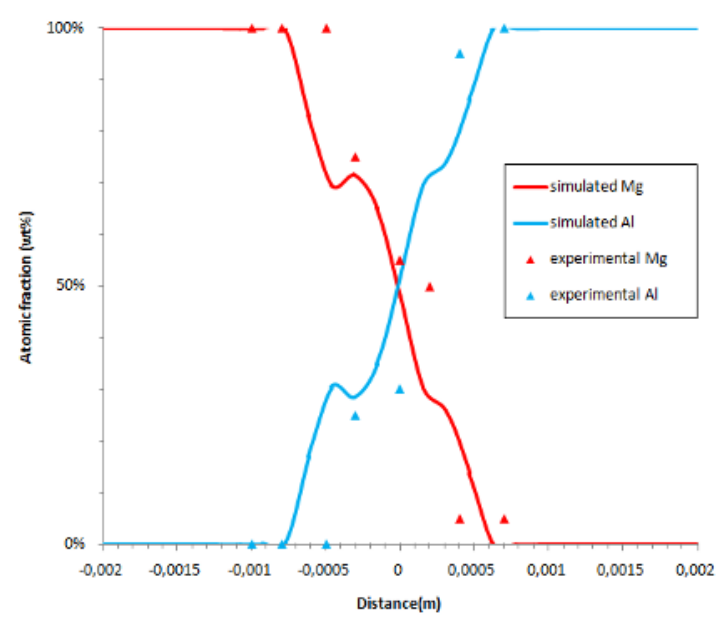

Fig. 3. Distribution of chemical elements at the bottom of the weld.

It can be seen from Figure 4 that the hardness increases considerably at the bottom and at the top of the bead. It is in the range of 220-240HV. The increase in hardness in the molten zone compared to that of the base metal shows the presence of intermetallic compounds. These intermetallic compounds are characterized by a high hardness. 


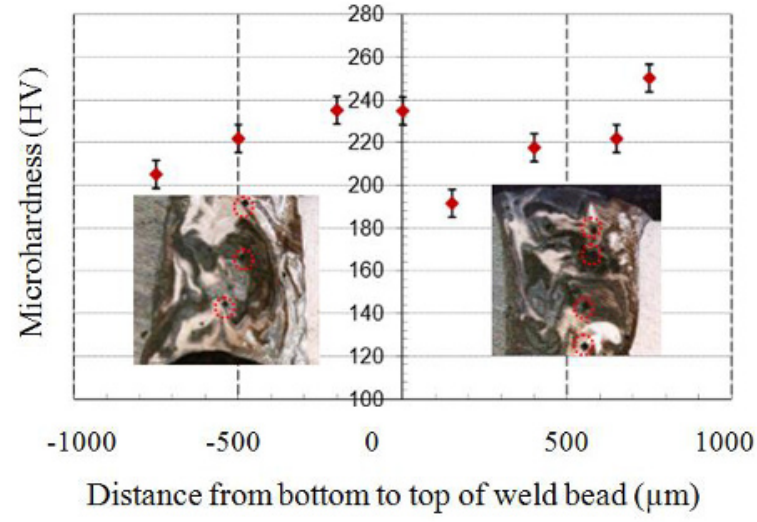

Fig. 4. Vickers Microhardness profile from the top of the weld bead to the bottom.

The formation of intermetallic compounds (IMCs) within the laser joint have been experimentally and numerically analyzed. At the top of the weld bead (area $\mathrm{M})$, the mass percentages given by the experiment are $28.25 \% \mathrm{Al}$ and $70.8 \% \mathrm{Mg}$. The chemical compositions of $\mathrm{Al}$ and $\mathrm{Mg}$ obtained numerically are $30 \%$ and $70 \%$ ,respectively. Thus, these percentages correspond to the mixture of a primary phase $(\alpha-\mathrm{Mg})$ and an intermetallic phase $\left(\mathrm{Al}_{12} \mathrm{Mg}_{17}\right)$. In the $\mathrm{N}$ area, the $\mathrm{Al}$ and $\mathrm{Mg}$ contents obtained by the numerical model at the bottom of the weld bead are $62 \%$ et $38 \%$ respectively. The mass percentages given by the experiment are $61.3 \%$ aluminum and $38.36 \%$ magnesium. This zone corresponds to the $\mathrm{Al}_{3} \mathrm{Mg}_{2}$ phase. The numerical results are in good agreement with the experimental results.

(a)

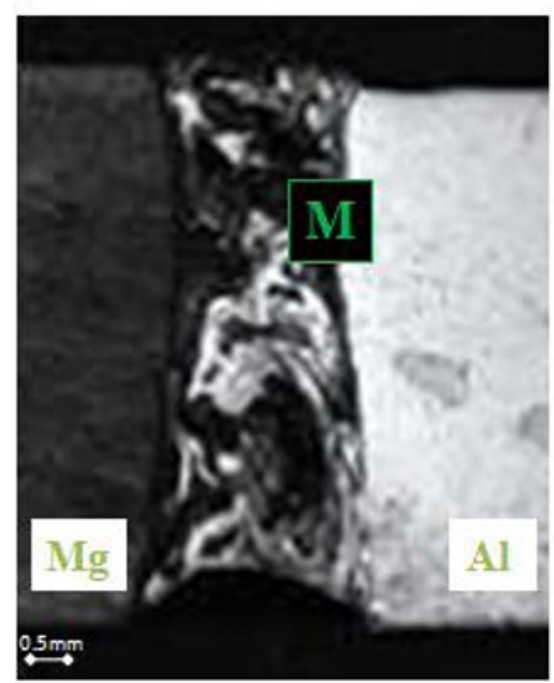

(b)

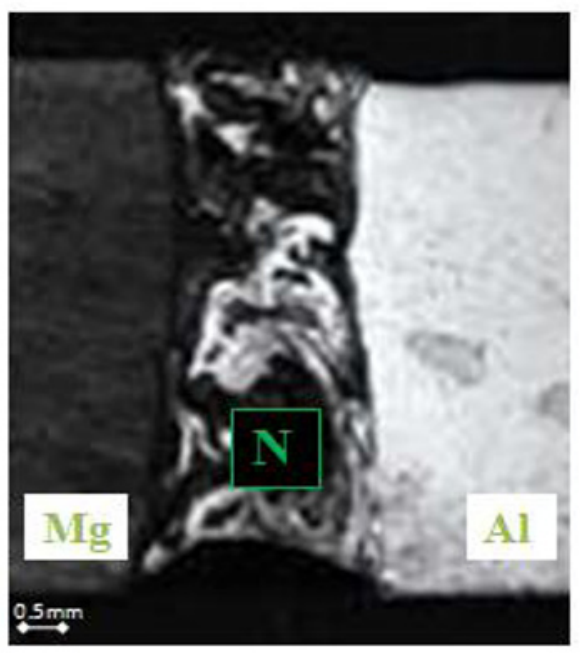

Fig. 5. Formation of intermetallic phases at (a) the top of the weld (b) the bottom of the weld.

\section{Conclusion}

In the present work, Aluminum / Magnesium dissimilar metals laser has been carried. A multi-physics computational model is utilized in order to analyze the molten pool behavior and weld bead formation. The experimental and numerical results showed that the brittle interface layer was composed mainly of the intermetallic compounds $\mathrm{Al}_{3} \mathrm{Mg}_{2}$ and $\mathrm{Al}_{12} \mathrm{Mg}_{17}$, which is the main cause of the weld cracking.

\section{References}

1. Z. Sun, JC. Ion, J. Mater. Sci., 30 (1995).

2. M. Dehghani, A. Amadeh and S. Akbari Mousavi, Mater Des. 49 (2013).

3. S. Chen, L. Li, Y. Chen, J. Huanga, J. Alloys Compd. (2011).

4. G. R. Mohammed, M. Ishak, S. Ahmad and H.A Abdulhadi, Metals, 7 (2017).

5. Z. Liang, G. Qin, L. Wang, X. Meng, F. Li, Mater. Sci. Eng. A (2015).

6. G. Virgil, R. Irinel, P. Alin, Adv. Mater. Res. 1029 (2014).

7. S. Bannour, M. Autric, J-E Masse, S Mattei, H Mhiri, Microstructure and properties of weldsbetween $5754 \mathrm{Al}$ alloys and AZ31 Mg alloys using a Yb:YAG laser, 20th International Symposium on High Power Laser Systems\& Applications, Chengdu, Sichuan Province, China (2014).

8. C.T. Chi, C.G. Chao, T.F. Liu, C.H. Lee, Scr. Mater. 56 (2007). 